\title{
Comparison of the efficacy of honey gel and clotrimazole cream in the treatment of vaginal candidiasis symptoms: a randomized clinical trial
}

Zahra Seifinadergoli ${ }^{1}$, Fatemeh Nahidi ${ }^{2}$, Abdolrasoul Safaiyan ${ }^{3}$, Yousef Javadzadeh ${ }^{4}$, Tahereh Eteraf-Oskouei ${ }^{5}$

${ }^{1}$ Department of Midwifery \& Reproductive Health, School of Nursing \& Midwifery, Shahid Beheshti University of Medical Sciences, Tehran, Iran

${ }^{2}$ Midwifery and Reproductive Health Research Center, Department of Midwifery \& Reproductive Health, School of Nursing \& Midwifery, Shahid Beheshti University of Medical Sciences, Tehran, Iran

${ }^{3}$ Assistant Professor, Road Traffic Center and Department of Biostatistical and Epidemiology, Tabriz Medical Science University, Tabriz, Iran

${ }^{4}$ Professor, Biotechnology Research Center and Faculty of Pharmacy, Tabriz University of Medical Sciences, Tabriz, Iran

${ }^{5}$ Associate Professor, Department Of Pharmacology and Toxicology, Faculty of Pharmacy, Tabriz University of Medical Sciences, Tabriz, Iran

\section{Type of article: Original}

\begin{abstract}
Background: Reproductive tract infection is a global health problem among women, particularly in East Asia. Accordingly, researchers are seeking drugs with fewer side effects, compared to chemical agents. In this regard, the antifungal properties of honey have been confirmed.

Objective: This study was conducted to examine the effects of vaginal honey gel and clotrimazole cream on symptoms of candidiasis in 2014-2015.

Methods: This single-blind clinical trial was conducted on 106 patients with clinical complaints and positive cultures. The study was conducted in Tabriz clinics and health centers during 2014-2015. The participants were randomly divided into clotrimazole cream and honey gel groups (n, 53 per group) and were assessed both pretreatment and post treatment (before, 4 and 8 days after treatment). The results were analyzed and compared in IBM-SPSS version 22, using McNemar's test, Cochran's Q test, generalized mixed model, and independentsamples t-test. The significance level was set at 0.05 .

Results: At all 3 time intervals, significant reductions were observed in vaginal symptoms, compared to pretreatment due to the use of honey gel and clotrimazole cream $(\mathrm{p}<0.001)$. On the other hand, the culture results were similar, and there was no significant difference between the groups $(\mathrm{p}>0.05)$.

Conclusion: Honey was effective in the treatment of candidiasis symptoms and could be used as an alternative or adjuvant for other antifungal drugs in the treatment of vaginal candidiasis symptoms.

Clinical Trial Registration: This study was registered in the Iranian Registry of Clinical Trials (http://www.irct.ir) with the registration code IRCT201604144317N9.

Funding: The authors received no financial support for the research.

Keywords: Honey; Clotrimazole; Vulvovaginal candidiasis; Symptom
\end{abstract}

\section{Corresponding author:}

Assistant Professor Dr. Fatemeh Nahidi, Midwifery and Reproductive Health Research Center, Department of Midwifery \& Reproductive Health, School of Nursing \& Midwifery, Shahid Beheshti University of Medical Sciences, Tehran, Iran.

Tel:+98.2188202512 Fax:+98.2188202512, Email: nahidi@sbmu.ac.ir.com and f.nahidi87@gmail.com

Received: April 09, 2017, Accepted: December 14, 2017, Published: June 2018

iThenticate screening: September 18, 2017, English editing: April 13, 2018, Quality control: April 15, 2018

This article has been reviewed / commented by four experts

Ethics approval: $1000 / 2577$

(C) 2018 The Authors. This is an open access article under the terms of the Creative Commons Attribution-NonCommercialNoDerivs License, which permits use and distribution in any medium, provided the original work is properly cited, the use is non-commercial and no modifications or adaptations are made. 


\section{Introduction}

Reproductive tract infection is a global health problem among women, particularly in East Asia (1). Women from any race and at any age may be infected with vulvovaginal candidiasis, as one of the most recurrent diseases around the world (2). This infection is responsible for nearly 13 million cases of vaginitis in women in North America. Moreover, 64 million women at reproductive age are infected in Brazil (3). The predisposing factors include pregnancy, use of high-dose oral estrogen or contraceptives, diabetes, use of broad-spectrum antibiotics, corticosteroids, immune cell deficiency, and obesity. Infection of the vagina or vulva may be as sociated with symptoms, such as severe itching, burning, pain, and white or whitish cottage cheese-like secretions (4-7). Discharge may be aqueous to thick and homogenous; in addition, there is a risk of dyspareunia $(5,8)$. Although this infection is rarely life threatening, it usually produces symptoms, such as sexual problems, vaginal dryness, and pain; moreover, it is associated with high costs of treatment $(4,9,10)$. In $80 \%$ to $90 \%$ of patients, clotrimazole eliminates the symptoms within 2 to 3 days, and produces negative culture results. The treatment agents include butoconazole, clotrimazole, miconazole, and terconazole (5, 11-13). Problems, such as limited variety of antifungal drugs, toxicity for body cells, reduced sensitivity of some Candida species to drugs, and disease recurrence, are the main concerns in the treatment of such diseases. Accordingly, researchers' attention has been drawn to new nonchemical drugs $(14,15)$. In fact, the search for new antifungal agents has prompted research on new chemotherapeutic agents from natural products. Since ancient times, honey has been used for the treatment of several diseases (16-18). It has been traditionally used in the treatment of ulcers, bedsores, and infections resulting from burns and wounds. Honey has been also found to be effective against organisms isolated from urinary tract infections (19-21). S.E. Blair argues that major antimicrobial factors in honey can help alleviate some antibioticresistant pathogens during wound care (22). Although several in vitro studies have demonstrated the antibacterial properties of honey, only a few have examined its activities against fungal species (23). Clinical studies have used only parts of honey, such as propolis, and have reported a reduction in colony count (24). In a study conducted on 129 women with vulvovaginitis candidiasis, honey combined with yoghurt was more effective in the treatment of clinical symptoms, compared to azole compositions (25). Mercan et al. conducted an in vitro study on the antimicrobial effects of honey in different parts of Turkey. Their results showed that different types of honey could inhibit bacterial growth (26). Honey also showed positive healing effects in combination with other substances, such as starch, olive oil, beeswax, and yoghurt $(25,27,28)$. It is highly important to choose a treatment, which can shorten the healing period and reduce the rate of recurrence, based on clinical and microscopic results. The effects of pure honey on Candida albicans have been only studied in vitro, and not in clinical settings. Therefore, considering the side-effects of chemical drugs and resistance against antifungal drugs in many patients (13, 29), this study was conducted to examine the efficacy of vaginal honey gel and clotrimazole cream in the treatment of vaginal candidiasis symptoms and to find an effective agent with fewer side-effects.

\section{Material and Methods}

\subsection{Trial design}

This randomized clinical trial was conducted to compare the effects of vaginal honey gel and clotrimazole cream on the symptoms of vulvovaginal candidiasis in Tabriz clinics and health centers during 2014-2015. The sample size was calculated to be 106 , based on previous studies. By assuming a power of $95 \%$ and confidence level of $95 \%$, the sample size was measured using the following formula:

$\mathrm{n}=\left(\mathrm{Z}_{1-\alpha / 2}+\mathrm{Z}_{1-\beta}\right)^{2} \times\left(\left(\mathrm{P}_{1}\left(1-\mathrm{P}_{1}\right)+\mathrm{P}_{2}\left(1-\mathrm{P}_{2}\right)\right) /\left(\mathrm{P}_{1}-\mathrm{P}_{2}\right)^{2}\right.$

Where: $\mathrm{n}$ is the sample size, $\mathrm{Z}_{1-\alpha / 2}$ equals $1.96, \mathrm{Z}_{1-\beta}$ equals 1.64 (test power, $95 \%$ ), and $\mathrm{P}$ represents the probability of the main outcome.

\subsection{Study population}

The research hypothesis was that vaginal honey gel is as effective as vaginal clotrimazole cream in the treatment of candidiasis symptoms. The statistical population consisted of women with vaginal discharge, vaginal itching or burning, dyspareunia, and urinary tract problems, who were referred to the selected health centers. A total of 300 potential patients were recruited in the study, out of which 190 patients were eliminated. Finally, 110 patients with positive culture and clinical symptoms were randomly divided into honey and clotrimazole groups (Figure 1). Four participants ( 2 from the study group and 2 from the control group) exited the study due to lack of follow-up. Consequently, the study was performed on a total of 106 patients (53 per group).

\subsection{Inclusion criteria}

The inclusion criteria were as follows: 1) age range, 18-45 years; 2) symptoms of vaginal candidiasis, based on interviews, observation, and laboratory studies; 3) non-pregnant women who had not used any immune suppressors 
or broad-spectrum antibiotics in the past month; and 4) no history of epilepsy, diabetes, cardiac diseases, hypertension, thyroid diseases, or advanced arthritis. On the other hand, the exclusion criteria were: 1) pregnancy during the study; 2) unwillingness to continue participation in the study; 3) necessity to use antibiotics during the study; 4) allergy to medicines; and 5) failure to follow the instructions (negligent use of drugs for more than one night).

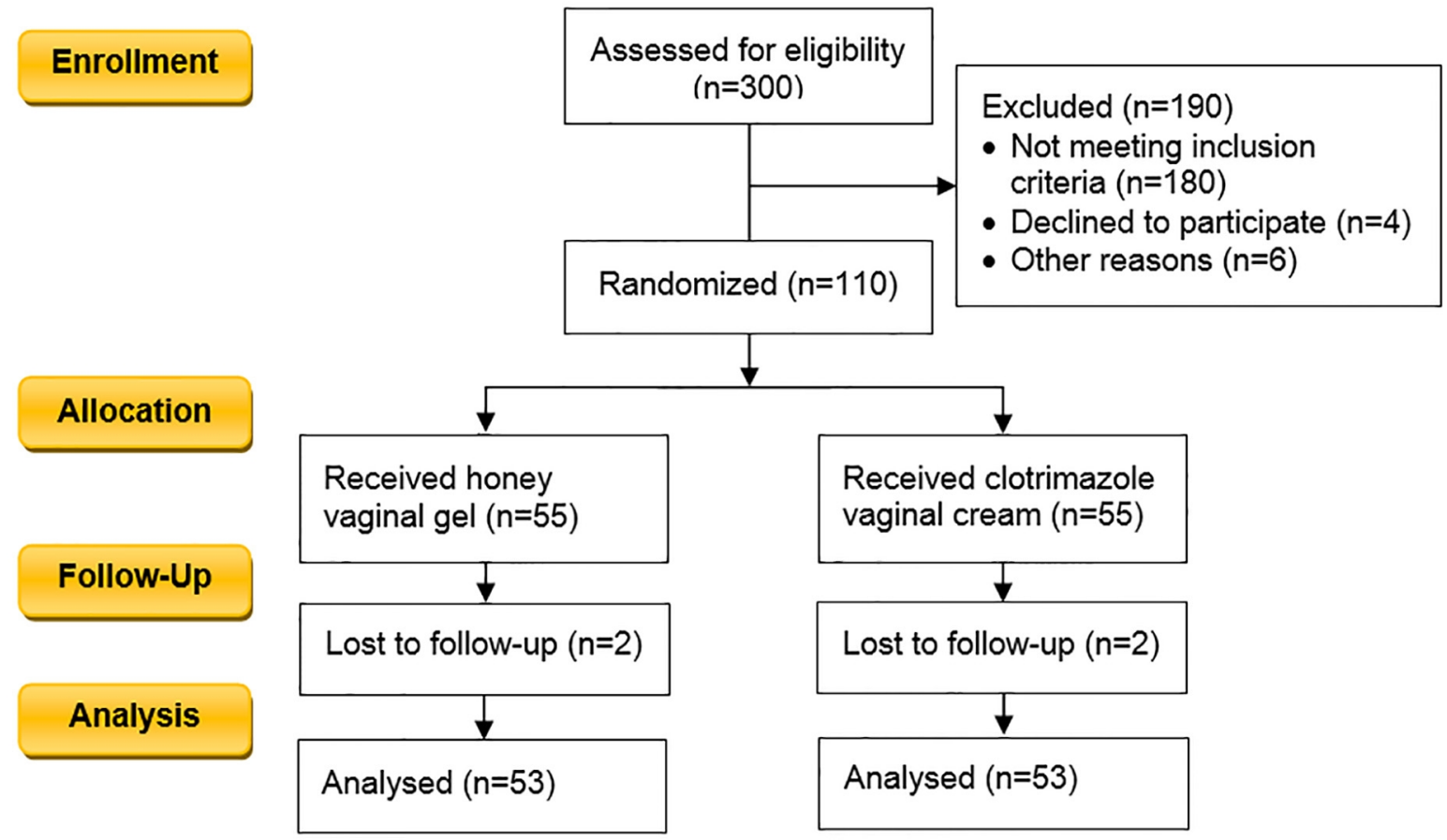

Figure 1. CONSORT 2010 Flow Diagram of the study

\subsection{Outcomes and instruments}

This study was performed on 106 participants, who were assigned to the honey and clotrimazole groups. The primary outcomes showed a reduction in all symptoms, including vaginal discharge, itching, burning, dyspareunia, and urinary tract problems after treatment. A questionnaire was used to collect the data. In this study, clotrimazole 1\% cream (Raha Company; registration number, 1228101540) and vaginal 50\% honey gel were used. Moreover, a microscope (model cx31 Sn-5mo9248, Olympus Corporation, Tokyo, Japan), a Chinese pH measurement paper (Q/CHSC1544-1999), Sabouraud Dextrose Agar (Paris, France), a glass tube (0.16×16), and KOH 10\% solution were used for the experiments. The questionnaires were completed by the researcher. To avoid biased information, the patients were unaware of the type of intervention. The ethical regulations of Shahid Beheshti University of Tehran and Tabriz University of Medical Sciences (ethical code, 1000/2577) were strictly observed. The demographic information, including age, family size, socioeconomic status, education, and occupation, was collected using a questionnaire. The researcher was trained by a laboratory technician on how to perform culture studies and interpret the results over 2 months. All 300 patients signed the informed consent forms. The honey concentration was determined at $30 \%$, based on gel stability.

\subsection{Interventions}

In this study, the honey used was produced by bees feeding on spring pollen in Sabalan Mountains around Ardebil city, whose purity and microbial tests had been determined by experts from Tabriz Agricultural Jihad. All formulation stages of honey gel were performed in the laboratory by a Professor of Pharmaceutics of Tabriz School of Pharmacy. The honey used contained $0.83 \%$ of saccharose, which complied with the international standards (maximum 5\%), with fructose to glucose ratio of 1.78 , which was normal compared to standard value (minimum 0.9). This honey also had an acceptable level of microbes. To prepare vaginal honey gel $50 \%$, first, preserved water was prepared using methyl paraben $2 \%$ and propyl paraben $0.02 \%$, and $1 \%$ of carbomer 934 was added to some of this water, and gently stirred until fully dissolved. Next, right amounts of honey were gently added, and after full dispersion, the remaining water was added and stirred. The final gel was produced by addition of tri-ethanol-amine 
in drops. The prepared honey gel was poured into unlabeled sterile tubes using a filling device. Clotrimazole $1 \%$ cream was also poured into similar sterile tubes. These tubes were coded A and B by the pharmaceutics professor. Demographic information, including information related to age, family size, socioeconomic status, education, and occupation, was collected through a questionnaire. The researcher was taught how to perform culture and interpret results by a laboratory technician over two months. Concentration of honey was determined as $30 \%$, based on stability of the gel. To observe ethical considerations, and because honey gel was being used for the first time on human mucosa, it was initially tested on 30 samples with positive candidiasis in a pilot study. Patients recovered from clinical symptoms after 8 days; yet, the majority still showed positive cultures. Hence, study was performed with increased concentration of honey to $50 \%$. Participants were provided with explanations about the study, then, they were examined, and samples were taken from them. Those with symptoms of vaginitis that met inclusion criteria completed a preliminary questionnaire. A disposable speculum was used without lubricant to take samples, and symptoms of disease were recorded in an observation checklist. Samples of vaginal discharge were taken using two sterile cotton swabs. The first swab was smeared over two slides; 1-2 drops of normal saline were poured on the first, and $\mathrm{KOH} 10 \%$ solution was poured on the second. The first slide was examined under a microscope at $10 \mathrm{X}$ magnification. Observation of key cells or flagellate parasites, and also amine odor in a whiff test meant diagnosis of Gardnerella or Trichomonas, and exclusion from study. The second slide was also examined under the same microscope, and observation of mycelium and blastospores meant positive smear test for candida. Next, vaginal discharge was assessed using $\mathrm{pH}$ paper, and $\mathrm{pH}$ less than 4-4.5, which confirmed candida infection was recorded in an observation checklist. Kappa coefficient of agreement was calculated for all cases (minimum agreement coefficient was 0.8 ). Otherwise, the sample was excluded from study with mixed infection diagnosis. The second swab showing positive smear for candida was sent to the laboratory in a tube containing normal saline to be transferred to fungi culture medium of Dextrose Agar for final diagnosis of candida species. Culture medium was prepared by the researcher according to manufacturers' instructions, and in sterile conditions, $20 \mathrm{~mL}$ was poured onto each 8-mm sterile plate. Afterward, the researcher prepared streak culture of the samples on culture medium. Samples cultured on Dextrose Agar were incubated at $30-37{ }^{\circ} \mathrm{C}$ for 48 to 72 hours, and colonies were examined under microscope after 72 hours by the researcher, supervised by a laboratory technician. Next, positive cultures for fungi were removed and poured into tubes containing human serum using a loop; in case of growth, presence of Candida albicans was confirmed. All species of candida were included in the study. Samples were then randomly coded, using randomization software. Participants were divided into honey gel and clotrimazole cream groups. Patients received 5 grams of $50 \%$ honey gel and 5 grams of $1 \%$ clotrimazole cream using an applicator every night for 8 nights. Samples were unaware of the content of the packages. All patients were provided with instructions of use and hygiene recommendations. They were also advised not to have sex without condoms, and avoid vaginal showers, use of other vaginal medications and antibiotics. Patients with confirmed test results showing fungal growth on culture medium and confirmed candida were contacted and asked to visit the clinic four days after commencement of use of medication. Patients' compliance with hygiene instructions and any serious complication were assessed.

\subsection{Follow-up}

In the follow-up stage after completion of treatment, patients were examined in terms of microscopic and other symptoms, and results were recorded in the observation checklist. Then, a second culture was prepared to ensure patients' recovery or otherwise, and results were recorded in the observation checklist. Data were analyzed using McNemar's test, Cochran Q, general mix model and independent $t$ tests using SPSS 22 statistical software and $\mathrm{p}<$ 0.05 was considered as significant level. If treatment in the honey gel group failed, patients were requested to attend the clinic the following day to receive routine treatment with clotrimazole cream for 8 days.

\subsection{Randomization and blinding}

Single-blinded method was used for blinding and hundred and six subjects with positive culture were divided into two groups (53) using NCSS PASS V11 software.

\subsection{Statistical methods}

The data were collected at 3 time intervals (baseline, 4 days after the intervention, and 8 days after the intervention) and analyzed in IBM( $)$ SPSS $\odot$ Statistics version 22 (IBMC Corp., Armonk, NY, USA). Descriptive (mean and standard deviation) and inferential statistics were calculated, using McNemar's and Cochran's Q test (for intragroup comparisons), generalized mixed model (comparison of two methods), and independent-samples t-test (for comparison of demographic parameters before the intervention). P-value less than 0.05 was considered statistically significant. 


\subsection{Ethical considerations}

All 300 patients participating in this study signed the informed consents. The ethical regulations dictated in the act provided by Shahid Beheshti University of Medical Sciences and Tabriz University of Medical Sciences (ref. no. of the ethics approval from Shahid Beheshti University of Medical Sciences: 1000/2577, date: January 05, 2015) were strictly observed. This study was registered in the Iranian Registry of Clinical Trials (http://www.irct.ir) with the registration code IRCT201604144317N9.

\section{Results}

No significant difference was found between honey and clotrimazole groups in terms of quantitative demographic parameters such as age, spouse's age, household size, gravida, parity, live births, frequency of sex per week, weight, BMI, and qualitative demographic parameters such as mother's education, father's education, house ownership, alcohol and cigarette use, cesarean section or miscarriage, menstruation, and previous history of infection. IUD was used as a contraceptive in $60 \%$ of patients in the honey group and $74 \%$ in the clotrimazole group. According to Chisquare test, the two groups were not significantly different in terms of type of contraception method $(\mathrm{p}<0.001)$. Table 1 shows the effects of honey gel and clotrimazole cream on symptoms of vaginal candidiasis on the three occasions: before treatment, 4 days and after completion of treatment in women with vaginal candidiasis. Comparing frequency of differences in symptoms before and 4 days after treatment showed no difference between the two groups in vaginal discharge. However, itching, burning, dyspareunia, and urinary problems had significantly reduced in both honey gel and clotrimazole cream groups $(\mathrm{p}<0.001)$. Comparing differences in symptoms 4 days after treatment and after completion of treatment, showed a reduction in vaginal discharge in both groups. Itching had not changed in the honey gel group, but reduced in the clotrimazole group. No difference was observed between the two groups in burning and dyspareunia or in urinary problems $(\mathrm{p}<0.001)$ (Table 1). Comparing differences in symptoms before treatment and after completion of treatment showed a reduction in all symptoms, including itching, burning, dyspareunia, and urinary problems after completion of treatment $(p<0.001)$ (Table 1). On the three occasions studied, the Cochran test showed a reduction in discharge, itching, burning, dyspareunia and urinary problems in both groups $(\mathrm{p}<0.001)$. According to the Mix Model test, the two groups were the same in terms of reduction in discharge, burning, and dyspareunia $(p>0.05)$ However, honey gel and clotrimazole had different effects on itching and urinary problems $(\mathrm{p}<0.001)$ (Table 1). A McNemar test showed a significant reduction in frequency of culture medium (Sabouraud Dextrose Agar) due to application of honey and clotrimazole after treatment $(\mathrm{p}<0.001)$. The Mix Model showed no significant difference between the two groups, which meant honey and clotrimazole had equal effects in turning positive culture into a negative one $(\mathrm{p}>0.05)$ (Table 2$)$.

Table 1. Differences between honey gel and clotrimazole cream in their effects on symptoms

\begin{tabular}{|c|c|c|c|c|}
\hline \multirow[t]{2}{*}{ Symptoms } & \multirow[t]{2}{*}{ Time } & \multicolumn{2}{|l|}{ Group } & \multirow{2}{*}{ p-value (Cochran Q test) } \\
\hline & & Honey vaginal gel & Clotrimazole vaginal cream & \\
\hline \multirow[t]{3}{*}{ Vaginal discharge } & Before treatment & $53(100 \%)$ & $53(100 \%)$ & \multirow[t]{3}{*}{$<0.001$} \\
\hline & Forth day & $52(98 \%)$ & $53(100 \%)$ & \\
\hline & Eighth day & $36(68 \%)$ & $38(72 \%)$ & \\
\hline \multirow[t]{3}{*}{ Itching } & Before treatment & $53(100 \%)$ & $53(100 \%)$ & \multirow[t]{3}{*}{$<0.001$} \\
\hline & Forth day & $3(10 \%)$ & $22(40 \%)$ & \\
\hline & Eighth day & $2(4 \%)$ & $7(10 \%)$ & \\
\hline \multirow[t]{3}{*}{ Burning } & Before treatment & $19(40 \%)$ & $27(50 \%)$ & \multirow[t]{3}{*}{$<0.001$} \\
\hline & Forth day & $0(0 \%)$ & $5(10 \%)$ & \\
\hline & Eighth day & $2(4 \%)$ & $0(0 \%)$ & \\
\hline \multirow[t]{3}{*}{ Dispareunia } & Before treatment & $37(70 \%)$ & $28(53 \%)$ & \multirow[t]{3}{*}{$<0.001$} \\
\hline & Forth day & $1(2 \%)$ & $4(8 \%)$ & \\
\hline & Eighth day & $1(2 \%)$ & $2(4 \%)$ & \\
\hline \multirow[t]{3}{*}{ Urinary problem } & Before treatment & $26(49 \%)$ & $16(30 \%)$ & \multirow[t]{3}{*}{$<0.001$} \\
\hline & Forth day & $8(15 \%)$ & $1(2 \%)$ & \\
\hline & Eighth day & $3(6 \%)$ & $0(0 \%)$ & \\
\hline
\end{tabular}


Table 2. Frequency distribution and comparison of culture media (dextrose agar) in honey gel and clotrimazole cream groups before treatment and after completion of treatment

\begin{tabular}{|l|l|l|l|l|l|}
\hline \multicolumn{2}{|l|}{ Treatment group } & Honey & \multicolumn{2}{l|}{ Clotrimazole } \\
\hline \multicolumn{2}{|l|}{ Time } & Day 1 & Day 8 & Day 1 & Day 8 \\
\hline Sabouraud dextrose agar & Negative & $0(0 \%)$ & $26(50 \%)$ & $0(0 \%)$ & $43(80 \%)$ \\
\cline { 2 - 6 } & Positive & $53(100 \%)$ & $27(50 \%)$ & $53(100 \%)$ & $10(20 \%)$ \\
\cline { 2 - 5 } & McNemar's test & $\mathrm{p}<0.001$ & $\mathrm{p}<0.001$ & \\
\cline { 2 - 5 } & Mix Model & $\begin{array}{l}\text { Disagreement between the two treatments }<<0.005 \\
\text { Disagreement of time between the two treatments } \mathrm{p}<0.001\end{array}$ \\
\hline
\end{tabular}

\section{Discussion}

Results of the current study indicated the antifungal effect of honey, like clotrimazole, on vaginitis symptoms. Similar results were also observed in a study by Darvishi et al., they compared the antifungal effect of honey plus yogurt with clotrimazole on vaginitis (4). Abdelmonem et al., also reported similar results regarding the antifungal effect of honey plus yogurt, compared with that of Tioconazole (25). Results of the current study indicated improvement in itching 8 days after the initiation of treatment in most of the subjects. In a study by Darvishi et al, improvement of itching was reported 7 days after the treatment in both groups of honey plus yogurt and clotrimazole (4). In a study by Abdelmonem et al., the itching was significantly improved after the completion of treatment course in the honey plus yogurt group compared with the Tioconazole group (25). However, results of a study by Fazel et al., showed improvement in itching in all subjects of the honey plus clotrimazole groups, while only $25 \%$ of the subjects in the clotrimazole group were improved (30); difference between the groups can be attributed to the antifungal activity of honey.

In the current study, the vaginal discharge significantly decreased in both honey and clotrimazole groups. For instance, in the study by Abdelmonem et al., (25) vaginal discharge reduced in both honey plus yogurt and clotrimazole groups. Reduced vaginal discharge was also reported in the study by Darvishi et al., who compared the antifungal activity of honey plus yogurt and clotrimazole (4). Similar results were also reported in a study by Ebrahimi et al., comparing antifungal activity of garlic and Fluconazole (1). In the current study, most of the subjects in both the honey plus yogurt and clotrimazole groups reported reduced vaginal burning that was similar to the results reported by Ebrahimi et al. (1), Darvishi et al. (4), and Abdelmonem et al. (25). In a study of dispareunia, most of the subjects in the honey and clotrimazole groups had improved which was similar to results reported by Ebrahimi et al. (1) who studied the antifungal activity of garlic and Fluconazole.

Results of the current study showed that urinary problems improved in most of the cases in the honey and clotrimazole groups, which was consistent with the results of Ebrahimi et al. (1), and Darvishi et al. (4); similar results can be attributed to anti-inflammatory and antifungal activities of honey. In the current study, most of the subjects were culture-negative in the honey group, similar to that of the clotrimazole group (25). In addition, results of the study by Ebrahimi et al., showed significant reduction in the number of patients with positive culture in both the garlic and fluconazole groups (1); similar to the study by Kordi et al., who compared the results of the garlic and clotrimazole groups (31). In an in vitro study by Al-Waili et al., honey inhibited the growth of fungi in agar plates (29); while it had lower antifungal effects in combination with olive oil and bees wax. Differences between the antifungal effect of the honey and honey plus olive oil plus bees wax groups can be explained by reduced antifungal activity of honey in combination with other materials. Vaginal burning and discharge were the main complaints of the patients in the current study that was similar to those of Ebrahimi et al. (1) and Grigoriou et al (32). In a study by Ahmad A. Khan, vaginal burning was the main complaint of the patients (33). Differences among the aforementioned studies in the frequency of symptoms can be explained by immune system differences of the subjects and the type of fungal infection. Evaluation and comparison of all symptoms were the main strengths of the current study. Short-term follow-up and differences in the immune system of the patients were among the limitations of the present study.

\section{Conclusions}

In this single-blinded, randomized clinical trial, honey and clotrimazole had similar reducing effects on vaginal discharge, burning, and dyspareunia, whereas they showed dissimilar effects on itching and urinary symptoms. They were both equally effective in making the culture and wet smear results negative. Therefore, honey can be suggested as an alternative or adjuvant for other antifungal medications in the treatment of symptoms of vaginal candidacies. 
http://www.ephysician.ir

Although several clinical trials have confirmed the therapeutic efficacy of honey, further research with a larger sample size is recommended to confirm the findings.

\section{Acknowledgments:}

This article was extracted from a research project, submitted to Shahid Beheshti University of Medical Sciences. We wish to express our gratitude to the education deputy and authorities of the international branch of Shahid Beheshti University of Medical Sciences and Tabriz University of Medical Sciences for supporting this project. We also would like to thank all the personnel of Shahryar Health Center in Tabriz, Iran, especially Ms. Ezadi, as well as the personnel of Zakaria Hospital laboratories, especially Ms. Asadollahzadeh for their assistance. We also thank all the participating patients for their cooperation.

\section{Clinical Trial Registration:}

This study was registered in the Iranian Registry of Clinical Trials (http://www.irct.ir), with the registration code, IRCT201604144317N9.

\section{Funding:}

The authors received no financial support for the research, authorship, and/or publication of this article.

\section{Conflict of Interest:}

There is no conflict of interest to be declared.

\section{Authors' contributions:}

All authors contributed to this project and article equally. All authors read and approved the final manuscript.

\section{References:}

1) Ebrahimy F, Dolatian M, Moatar F, Majd HA. Comparison of the therapeutic effects of Garcin and fluconazole on Candida vaginitis. Singapore Med J. 2015; 56(10): 567-72. doi: 10.11622/smedj.2015153. PMID: 26512149, PMCID: PMC4613933.

2) Babic M, Hukic M. Candida albicans and non-albicans species as etiological agent of vaginitis in pregnant and non-pregnant women. Bosn J Basic Med Sci. 2010; 10(1): 89-97. doi: 10.17305/bjbms.2010.2744. PMID: 20192939.

3) Achkar JM, Fries BC. Candida infections of the genitourinary tract. Clin Microbiol Rev. 2010; 23(2): $253-$ 73. doi: 10.1128/CMR.00076-09. PMID: 20375352, PMCID: PMC2863365.

4) Darvishi M, Jahdi F, Hamzegardeshi Z, Goodarzi S, Vahedi M. The Comparison of vaginal cream of mixing yogurt, honey and clotrimazole on symptoms of vaginal candidiasis. Glob J Health Sci. 2015; 3(7): 108-16. doi: 10.5539/gjhs.v7n6p108. PMID: 26153168, PMCID: PMC4803919.

5) Wilkins LW. Berek, Novak's Gynecology 15th Edition. Lippincott Williams and Wilkins; 2012: 1560. doi: 10.1007/s13224-014-0538-z.

6) N. GRSaDD. Danforth obstetrics and gynecology 2008. N Engl J Med. 1990; 323(7): 493-4. doi: 10.1056/NEJM199008163230723.

7) Banaeian-Borujeni S, Mobini GR, Pourgheysari B, Validi M. Comparison of the effect of honey and miconazole against Candida albicans in vitro. Advanced Biomedical Research. 2013; 2: 57 . doi: 10.4103/2277-9175.115800. PMID: 24223372, PMCID: PMC3814582.

8) Hezarjaribi HZ, Fakhar M, Shokri A, Teshnizi SH, Sadough A, Taghavi M. Trichomonas vaginalis infection among Iranian general population of women: a systematic review and meta-analysis. Parasitol res. 2015; 114(4): 1291-1300. doi: 10.1007/s00436-015-4393-3. PMID: 25732256.

9) $\mathrm{Hu} \mathrm{H1}$, Merenstein DJ, Wang C, Hamilton PR, Blackmon ML, Chen H, et al. Impact of eating probiotic yogurt on colonization by Candida species of the oral and vaginal mucosa in HIV-infected and HIVuninfected women. Mycopathologia. 2013; 179(3-4): 175-81. doi: 10.1007/s11046-013-9678-4. PMID: 23925786, PMCID: PMC3903393.

10) Marot-Leblond A, Nail-Billaud S, Pilon F, Beucher B, Poulain D, Robert R. Efficient diagnosis of vulvovaginal candidiasis by use of a new rapid immunochromatography test. Journal of clinical microbiology. 2009; 47(12): 3821-5. doi: 10.1128/JCM.01168-09.

11) Borisov I, Kolarov G, Bobcheva S, Ivanova A. Treatment of chronic recurrent vulvovaginal candidiasis with fluconazole (fungolon-Actavis). Akush Ginekol (Sofiia). 2005; 44(2): 17-20. PMID: 16028398.

12) Physical Anthropology. 2009; 58(2): 271-404. doi: 10.1177/000134550905800202. 
13) Andreas H, Groll SCP, Thomas J. Clinical Pharmacology of Systemic Antifungal Agents: A Comprehensive Review of Agents in Clinical Use, Current Investigational Compounds, and Putative Targets for Antifungal Drug Development. Elsevier. 1998. doi: 10.1016/s1054-3589(08)60129-5.

14) Katiraii F, Khosravii A. Comparation of MIC some essences of Iran domestic plant on growth candida spacies resister and sensitive for Azole Drugs. Journal drug plants. 2008; 7: 37-44.

15) Mahdavi S, Moliji Gh. Effect of Iran north Honey on candida albicans. Journal Babol university of medical sciences. 2008; 5: 15-22.

16) Koc AN, Silici S, Ercal BD, Kasap F, Hörmet-Oz HT, Mavus-Buldu H. Antifungal Activity of Turkish Honey against Candida spp. and Trichosporon spp: an in vitro evaluation. Med Mycol. 2009; 47(7): $707-$ 12. doi: 10.3109/13693780802572554. PMID: 19888803.

17) Eteraf-Oskouei T, Najafi M. Traditional and Modern Uses of Natural Honey in Human Diseases: A Review. Iran J Basic Med Sci. 2013; 16(6): 731-42. PMID: 23997898 , PMCID: PMC3758027.

18) Asadi-Pooya AA, Pnjehshahin MR, Beheshti S. The antimycobacterial effect of honey: an in vitro study. Riv biol. 2002; 96(3): 491-5. PMID: 15055885.

19) Theunissen F, Grobler S, Gedalia I. The antifungal action of three South African honeys on Candida albicans. Apidologie. 2001; 32(4): 371-9.

20) Al-Waili NS, Saloom KY. Effect of topical honey on post-operative wound infections due to gram positive and gram negative bacteria following caesarean sections and hysterectomies. Eur J Med Res. 1999; 4(3): 126-30. PMID: 10085281.

21) Al-Waili NS. Investigating the antimicrobial activity of natural honey and its effects on the pathogenic bacterial infections of surgical wounds and conjunctiva. J Med Food. 2004; 7: 210-22. doi: 10.1089/1096620041224139. PMID: 15298770.

22) Blair SE, Carter DA. The potential for honey in the management of wounds and infection. Australian Infection Control. 2005; 10(1): 24-31. doi: 10.1071/HI05024.

23) Irish J, Carter DA, Shokohi T, Blair SE. Honey has an antifungal effect against Candida species. Medical Mycology. 2006; 44(3): 289-91. doi: 10.1080/13693780500417037. PMID: 16702110.

24) Kacaniova M, Melich M, Knazovicka V, Felsociova S, Sudzinova J. The Antimicrobial Activity of Honey And Propolis Against Yeasts Candida Species. Zootehnie şi Biotehnologii. 2009; 42(2).

25) Abdelmonem AM, Rasheed SM, Mohamed AS. Bee-honey and yogurt: a novel mixture for treating patients with vulvovaginal candidiasis during pregnancy. Archives of gynecology and obstetrics. 2012; 286(1): 109-14. doi: 10.1007/s00404-012-2242-5. PMID: 22314434.

26) Mercan N, Guvensen A, Celik A, Katircioglu H. Antimicrobial activity and pollen composition of honey samples collected from different provinces in Turkey. Natural Product Research. 2007; 21(3): 187-95.

27) Boukraa L, Benbarek H, Moussa A. Synergistic action of starch and honey against Candida albicans in correlation with diastase number. Brazilian Journal of Microbiology. 2008; 39(1): 40-3. doi: 10.1590/S1517-83822008000100010. PMID: 24031175, PMCID: PMC3768358.

28) Boukraâ L, Bouchegrane S. Additive action of honey and starch against Candida albicans and Aspergillus niger. Rev Iberoam Micol. 2007; 24(4): 309-11. PMID: 18095766.

29) Al-Waili NS, Akmal M, Al-Waili FS, Saloom KY, Ali A. The antimicrobial potential of honey from United Arab Emirates on some microbial isolates. Med Sci Monit. 2005; 11: 433-8.

30) Fazel N, Hashemian M, Ramezani M, Akaberi A. Comparison of honey with clotrimazole alone and mixed on candidial vaginitis. Iranina Journal of Obstetrics Gynecology and Infertility. 2011; 14: 48-54.

31) Kordi M, Jahangiri N, Rakhshandeh $\mathrm{H}$, Gholami H. Comparison of the effect of garlic extract vaginal douche and clotrimazol vaginal cream in the treatment of women with vaginal candidiasis. IJOGI. 2005; 8: $33-42$.

32) Grigoriou O, Baka S, Makrakis E, Hassiakos D, Kapparos G, Kouskouni E. Prevalence of clinical vaginal candidiasis in a university hospital and possible risk factors. Eur J Obstet Gynecol Reprod Biol. 2006; 126 : 121-5. doi: 10.1016/j.ejogrb.2005.09.015. PMID: 16256258.

33) Ahmad A, Khan AU. Prevalence of candida species and potential risk factors for vulvovaginal candidiasis in Aligarh, India. Eur J Obstet Gynecol Reprod Biol. 2009; 144: 68-71. doi: 10.1016/j.ejogrb.2008.12.020. PMID: 19261369. 